A. Hayashimoto

Nagoya Math. J.

Vol. 150 (1998), 95-104

\title{
ON THE CLASSIFICATION THEOREM FOR CR MAPPINGS
}

\author{
ATSUSHI HAYASHIMOTO
}

\begin{abstract}
Let $f: M \rightarrow M^{\prime}$ be a real analytic CR mapping between hypersurfaces with $f(p)=q$, where $p \in M$ and $q \in M^{\prime}$. In this paper, the relation between the type at $p$ and the one at $q$ is considered. As a corollary of the type condition theorem (Theorem 1.1), a classification theorem, which states that under certain type condition, any real analytic CR mapping as above is constant, is proved.
\end{abstract}

\section{$\S 1$. Introduction}

The goal of this paper is to contribute to the following classification problem for CR mappings.

Problem. Classify $C R$ mappings between $C R$ manifolds in terms of CR geometry (for example, Levi form, type of point, minimality and so on).

Let $M$ be a generic CR submanifold (see $\S 2$ ) of $\mathbb{C}^{n}$ with $\operatorname{dim}_{\mathbb{R}} M=$ $2 n-d$. We shall prove that under certain type condition, any CR mapping between generic CR submanifolds is constant, which is a corollary to Theorem 4.1. In the following theorem, $M$ and $M^{\prime}$ are real analytic, generic CR submanifolds containing the origin as a point of type $\left(l_{1}, \ldots, l_{d}\right)$ and type $\left(l_{1}^{\prime}, \ldots, l_{d}^{\prime}\right)$, respectively (definition of type of point will be given in $\S 2$ ). After a suitable coordinate change, we may assume that, for a sufficiently small neighborhood $U$ of the origin, we have $\left(\{0\}^{n-d} \times \mathbb{R}^{d}\right) \cap U \subset M$ and the $s_{k}$ are real parts of the transversal coordinates (see $\left.\S 2\right)$. $(f, g)$ is a real analytic CR mapping with $(f, g)(0,0)=(0,0)$.

THEOREM 1.1. Let $M$ and $M^{\prime}$ be generic $C R$ submanifolds of $\mathbb{C}^{n}$ with $\operatorname{dim}_{\mathbb{R}} M=\operatorname{dim}_{\mathbb{R}} M^{\prime}=2 n-d$ and $(f, g)=\left(f_{1}, \ldots, f_{n-d}, g_{1}, \ldots, g_{d}\right):$ $M \rightarrow M^{\prime}$ a CR mapping satisfying $\operatorname{det}\left(\left(\partial g_{j} / \partial s_{k}\right)(0)\right)_{j, k=1, \ldots, d} \neq 0$. Assume that, for sufficiently small neighborhood $U$ of the origin, $(f, g)$ satisfies

Received October 23, 1996. 
$(f, g)\left(\left(\{0\}^{n-d} \times \mathbb{R}^{d}\right) \cap U\right) \subset\{0\}^{n-d} \times \mathbb{R}^{d}$. Then the type condition $l_{j} \geq l_{j}^{\prime}$ holds for $j=1, \ldots, d$.

In the previous paper [5], the author gave another proof of the Landucci Theorem [6] in CR geometric style, namely, by using the tangential CauchyRiemann operator. Theorem 1.1 is motivated by the Landucci Theorem and its proof in CR geometric style.

Next we consider an application of Theorem 1.1 to a classification theorem for CR mappings. There are some previous results which assert that if CR submanifolds satisfy certain conditions, then any CR mapping between them is constant. For example, M. S. Baouendi and L. P. Rothschild proved the following theorem.

Theorem. (M. S. Baouendi-L. P. Rothschild [2]) Let $M$ and $M^{\prime}$ be smooth hypersurfaces and $H$ a smooth $C R$ mapping between them. If $M$ and $M^{\prime}$ are of $D$-finite type at $p_{0}$ and $H\left(p_{0}\right)$ respectively, then either $H$ is constant or $\operatorname{Jac}(H) \not \equiv 0$.

We shall prove another type of theorem like this as a corollary of Theorem 4.1 as follows.

CoRollary. Let $M$ and $M^{\prime}$ be real analytic hypersurfaces in $\mathbb{C}^{2}$ with type $_{0} M=l$ and type $M^{\prime}=l^{\prime}$, respectively. If the type condition $l / l^{\prime} \notin \mathbb{N}$ holds, then any $C R$ mapping $(f, g): M \rightarrow M^{\prime}$ is constant.

We are interested in the property of $l_{j} / l_{j}^{\prime}$. In the case $n=2, d=1$ in Theorem 1.1, we have $l / l^{\prime} \in \mathbb{N}$ (Theorem 4.1). But in case $n \geq 3$, unlike $n=2$, it may occur that $l_{j} / l_{j}^{\prime} \notin \mathbb{N}$ even if $d=1$. In fact we shall give such a $\mathrm{CR}$ mapping and $\mathrm{CR}$ submanifolds in $\S 5$. In that case, we shall see that $l_{j} / l_{j}^{\prime}(>1)$ can take any rational number.

This paper is organized as follows. In $\S 2$, we give a definition of type of point and basic results on CR mappings. In $\S 3$, we prove Theorem 1.1. In $\S 4$, we consider the special case of Theorem $1.1, n=2, d=1$. In $\S 5$, we give an example as mentioned above.

I would like to express my heartfelt gratitude to Prof. Takeo Ohsawa for giving me the idea of the proof, which was originally used in my master thesis [5], and some useful advice during my preparing this paper. 


\section{§. Notation and basic results}

Let $M$ be a smooth submanifold of $\mathbb{C}^{n}$ with $\operatorname{dim}_{\mathbb{R}} M=2 n-d, 0 \leq d \leq$ $n$. We call $M$ a CR submanifold if the holomorphic tangent space of $M$ at $p$, denoted by $H_{p}(M)$, has constant dimension for any $p$. A CR submanifold $M$ is called generic if $\operatorname{dim}_{\mathbb{R}} H_{p}(M)$ is minimal $(=2 n-2 d)$. A smooth mapping between $\mathrm{CR}$ submanifolds is a $\mathrm{CR}$ mapping if its components are annihilated by the induced Cauchy-Riemann operators on the source CR submanifold. In this paper, we always assume that generic CR submanifolds are real analytic ones containing the origin and that $\mathrm{CR}$ functions or $\mathrm{CR}$ mappings are real analytic ones preserving the origin. We define $T_{p}^{\mathbb{C}}(M)$ as a complexification of the tangent space of $M$ at $p$ and $H^{\mathbb{C}}(M)$ as a complexification of the holomorphic tangent bundle of $M$. We shall define the vector subspace $\mathcal{L}_{p}^{k}(M)$ of $T_{p}^{\mathbb{C}}(M)$ for $k \geq 1$. By the Lie bracket of length $k$ at $p$ generated by $H^{\mathbb{C}}(M)$, we mean a differential operator of the form

$$
\left[L_{1},\left[L_{2}, \ldots,\left[L_{k-1}, L_{k}\right] \ldots\right]_{p}, L_{j} \in H^{\mathbb{C}}(M) .\right.
$$

Let $\mathcal{L}_{p}^{1}(M)$ be $H_{p}^{\mathbb{C}}(M)$. We define $\mathcal{L}_{p}^{k}(M)$ as the vector subspace of $T_{p}^{\mathbb{C}}(M)$ spanned (over $\mathbb{C}$ ) by $H_{p}^{\mathbb{C}}(M)$ and all Lie brackets of length $j(j \leq k)$ at $p$ generated by $H^{\mathbb{C}}(M)$. Then we have

$$
H_{p}^{\mathbb{C}}(M)=\mathcal{L}_{p}^{1}(M) \subset \mathcal{L}_{p}^{2}(M) \subset \ldots \subset T_{p}^{\mathbb{C}}(M) .
$$

Definition 2.1. ([3], [4]) Let $M$ be a generic CR submanifold with $\operatorname{dim}_{\mathbb{R}} M=2 n-d$. We say that a point $p \in M$ has type $\left(l_{1}, \ldots, l_{d}\right)$ if the following conditions hold.

1. $\operatorname{dim}_{\mathbb{C}} \mathcal{L}_{p}^{j}(M)=2 n-2 d \quad j<l_{1}$.

2. $\operatorname{dim}_{\mathbb{C}} \mathcal{L}_{p}^{j}(M)=2 n-2 d+i \quad l_{i} \leq j<l_{j+1}$.

3. $\operatorname{dim}_{\mathbb{C}} \mathcal{L}_{p}^{j}(M)=2 n-d \quad j \geq l_{d}$.

Denote by type ${ }_{p} M=\left(l_{1}, \ldots, l_{d}\right)$. In this paper we assume $l_{1}<\ldots<l_{d}$.

We may assume that, after a suitable coordinate change, local defining functions for a generic $\mathrm{CR}$ submanifold $M$ with type ${ }_{0} M=\left(l_{1}, \ldots, l_{d}\right)$ have the forms

$$
\left\{\begin{array}{c}
r_{1}(z, w)=t_{1}-h_{1}(z, \bar{z}, s) \\
\vdots \\
r_{d}(z, w)=t_{d}-h_{d}(z, \bar{z}, s),
\end{array}\right.
$$


where $w=s+i t \in \mathbb{C}^{d}$ and

$$
h_{j}(z, \bar{z}, s)=\sum_{\substack{|\nu|+|\mu| \geq l_{j} \\|\nu|,|\mu| \geq 1,|\tau| \geq 0}} h_{\nu, \mu, \tau}^{j} z^{\nu} \bar{z}^{\mu} s^{\tau}
$$

is real analytic. In this representation, we call $w$ transversal coordinates. It follows from these expansions that, for a sufficiently small neighborhood $U$ of the origin, we have $\left(\{0\}^{n-d} \times \mathbb{R}^{d}\right) \cap U \subset M$. Notation for $M^{\prime}$ will be denoted by 'dash' style.

By the analogous argument of [1], any real analytic CR function $F$ : $M \rightarrow \mathbb{C}$ can be expressed as a power series, where $M$ is defined by $(2.1)$ and (2.2).

Lemma 2.1. ([1]) Let $M$ be a generic $C R$ submanifold and $F: M \rightarrow \mathbb{C}$ a real analytic $C R$ function. Then $F$ can be expressed as

$$
F(z, \bar{z}, s)=\sum_{|\alpha|+|p| \geq 1} A_{\alpha, p} z^{\alpha}(s+i h(z, \bar{z}, s))^{p} .
$$

Next we consider CR mappings between generic CR submanifolds defined by (2.1), (2.2) and their 'dash' style.

Lemma 2.2. Let $M$ and $M^{\prime}$ be generic $C R$ submanifolds and $(f, g)=$ $\left(f_{1}, \ldots, f_{n-d}, g_{1}, \ldots, g_{d}\right): M \rightarrow M^{\prime}$ a $C R$ mapping such that

$$
(f, g)\left(\left(\{0\}^{n-d} \times \mathbb{R}^{d}\right) \cap U\right) \subset\{0\}^{n-d} \times \mathbb{R}^{d}
$$

holds for sufficiently small neighborhood $U$ of the origin.

Then $f_{1}, \ldots, f_{n-d}, g_{1}, \ldots, g_{d}$ can be expressed as

$$
\begin{aligned}
& f_{j}(z, \bar{z}, s)=\sum_{|\alpha| \geq 1,|p| \geq 0} a_{\alpha, p}^{j} z^{\alpha}(s+i h)^{p}, \\
& g_{k}(z, \bar{z}, s)=\sum_{|q| \geq 1} b_{q}^{k}(s+i h)^{q}, \quad b_{q}^{k} \in \mathbb{R},
\end{aligned}
$$

for $j=1, \ldots, n-d, k=1, \ldots, d$.

Proof. By Lemma 2.1, $f_{j}$ and $g_{k}$ are expanded as

$$
f_{j}(z, \bar{z}, s)=\sum_{|\alpha|+|p| \geq 1} a_{\alpha, p}^{j} z^{\alpha}(s+i h(z, \bar{z}, s))^{p}
$$


and

$$
g_{k}(z, \bar{z}, s)=\sum_{|\beta|+|q| \geq 1} b_{\beta, q}^{k} z^{\beta}(s+i h(z, \bar{z}, s))^{q} .
$$

First, by (2.3), we obtain

$$
\begin{aligned}
& \sum_{|p| \geq 1} a_{0, p}^{j} s^{p}=0 \quad \text { for } j=1, \ldots, n-d, \\
& \quad \text { and } \quad \sum_{|q| \geq 1} b_{0, q}^{k} s^{p} \in \mathbb{R} \quad \text { for } k=1, \ldots, d,
\end{aligned}
$$

namely, $a_{0, p}^{j}=0$ for $|p| \geq 1$ and $b_{0, q}^{k} \in \mathbb{R}$ for $|q| \geq 1$. These give a desired expansion of $f$. Substitute these into expansions of $f$ and $g$. Then resulting expansions satisfy

$$
\begin{aligned}
& \frac{1}{2 i} \sum_{|\beta|+|q| \geq 1}\left[b_{\beta, q}^{k} z^{\beta}(s+i h)^{q}-\bar{b}_{\beta, q}^{k} \bar{z}^{\beta}(s-i h)^{q}\right] \\
&=\sum_{\substack{|\nu|+|\mu| \geq l_{k}^{\prime} \\
|\nu|,|\mu| \geq 1,|\tau| \geq 0}} h_{\nu, \mu, \tau}^{\prime k}\left(\sum_{|\alpha| \geq 1,|p| \geq 0} a_{\alpha, p} z^{\alpha}(s+i h)^{p}\right)^{\nu} \\
& \times\left(\sum_{\substack{|\alpha| \geq 1,|p| \geq 0 \\
a_{\alpha, p}}} \bar{z}^{\alpha}(s-i h)^{p}\right)^{\mu} \\
& \times\left(\frac{1}{2} \sum_{|\beta|+|q| \geq 1}\left[b_{\beta, q} z^{\beta}(s+i h)^{q}+\bar{b}_{\beta, q} \bar{z}^{\beta}(s-i h)^{q}\right]\right)^{\tau},
\end{aligned}
$$

for $k=1, \ldots, d$.

Picking up the sum of the terms that are not multiplies of $z_{i} \bar{z}_{j}$ from both sides of (2.4), we get

$$
\sum_{|\beta| \geq 1,|q| \geq 0}\left(b_{\beta, q}^{k} z^{\beta} s^{q}-\bar{b}_{\beta, q}^{k} \bar{z}^{\beta} s^{q}\right)=0 \quad \text { for } k=1, \ldots, d .
$$

Therefore we obtain $b_{\beta, q}^{k}=0$, for $k=1, \ldots, d,|\beta| \geq 1$ and $|q| \geq 0$, which implies that $g_{k}$ has the expansion,

$$
g_{k}(z, \bar{z}, s)=\sum_{|q| \geq 1} b_{0, q}^{k}(s+i h)^{q} .
$$

By replacing $b_{0, q}^{k}$ with $b_{q}^{k}$, we get the assertion of lemma. 
We need the following lemma to prove Theorem 4.1.

LEMMA 2.3. Let the notation be the same as in Lemma 2.2 with $n=2$, $d=1$. Then $g=0$ implies $f=0$.

Proof. Assume $g=0$. Then we have $h^{\prime}(f, \bar{f}, \operatorname{Re} g)=0$, namely,

$$
\begin{array}{r}
\sum_{\substack{\nu+\mu \geq l^{\prime} \\
\nu, \mu \geq 1, \tau \geq 0}} h_{\nu, \mu, \tau}^{\prime}\left(\sum_{\alpha \geq 1, p \geq 0} a_{\alpha, p} z^{\alpha}(s+i h)^{p}\right)^{\nu}\left(\sum_{\alpha \geq 1, p \geq 0} \bar{a}_{\alpha, p} \bar{z}^{\alpha}(s-i h)^{p}\right)^{\mu} \\
\times\left(\frac{1}{2} \sum_{q \geq 1}\left[b_{q}(s+i h)^{q}+\bar{b}_{q}(s-i h)^{q}\right]\right)^{\tau}=0
\end{array}
$$

Now consider the case $\tau=0$. Since, in the above equality, the sum of the terms of degree $l^{\prime}$ in $z$ and $\bar{z}$ satisfies

$$
\sum_{\substack{\nu+\mu=l^{\prime} \\ \nu, \mu \geq 1}} h_{\nu, \mu, 0}^{\prime}\left(\sum_{p \geq 0} a_{1, p} z s^{p}\right)^{\nu}\left(\sum_{p \geq 0} \bar{a}_{1, p} \bar{z} s^{p}\right)^{\mu}=0
$$

we get $a_{1, p}=0$ for $p \geq 0$. Substituting these into (2.5) and observing the terms of degree $2 l^{\prime}, 3 l^{\prime}, \ldots$ in the resulting equality repeatedly, we obtain $a_{\alpha, p}=0$ for any $\alpha$ and $p$, which implies the conclusion.

\section{$\S 3$. Proof of Theorem 1.1}

In this section, we prove Theorem 1.1. Theorem 1.1 is a generalization of the theorem the author gave in [5], where $M$ and $M^{\prime}$ are boundaries of pseudoellipsoids.

For each $j=1, \ldots, d$, we define $Q_{j}=\left\{\left(q_{1}, \ldots, q_{d}\right) \in \mathbb{Z}_{\geq 0}^{d} \mid q_{j} \geq 1\right\}$. Assume that the conclusion of Theorem 1.1 does not hold. Then there exists $j$ such that $l_{j}<l_{j}^{\prime}$. Put $j_{0}=\min \left\{j \mid l_{j}<l_{j}^{\prime}\right\}$. Then we have the inequality;

$$
l_{1}<\ldots<l_{j_{0}}<l_{j_{0}}^{\prime}<\ldots<l_{d}^{\prime}
$$

The CR mapping $(f, g)$ satisfies $\operatorname{Im} g_{j}=h^{\prime}(f, \bar{f}, \operatorname{Re} g)$, for $j=1, \ldots, d$, 
namely,

$$
\begin{aligned}
& \frac{1}{2 i} \sum_{|q| \geq 1} b_{q}^{j}\left[(s+i h)^{q}-(s-i h)^{q}\right] \\
&= \sum_{\substack{|\nu|+|\mu| \geq l_{j}^{\prime} \\
|\nu|,|\mu| \geq 1}} h_{\nu, \mu, 0}^{\prime}\left(\sum_{|\alpha| \geq 1,|p| \geq 0} a_{\alpha, p} z^{\alpha}(s+i h)^{p}\right)^{\nu} \\
& \times\left(\sum_{|\alpha| \geq 1,|p| \geq 0} \bar{a}_{\alpha, p} \bar{z}^{\alpha}(s-i h)^{p}\right)^{\mu}+\text { higher terms, } \\
& j=1, \ldots, d .
\end{aligned}
$$

The minimal degree of $z$ and $\bar{z}$ on the right hand side of (3.1) with $j=$ $j_{0}, \ldots, d$ equals to $l_{j_{0}}^{\prime}$. Thus picking up the terms of degree $l_{1}, \ldots, l_{j_{0}}\left(<l_{j_{0}}^{\prime}\right)$ from both sides of (3.1), we get

$$
\sum_{|q| \geq 1} b_{q}^{j} s_{1}^{q_{1}} \cdots s_{k}^{q_{k}-1} \cdots s_{d}^{q_{d}} h_{k}^{\left(l_{k}\right)}=0, \quad \text { for } j=j_{0}, \ldots, d, k=1, \ldots, j_{0}
$$

where $h_{k}^{\left(l_{k}\right)}$ stands for the homogeneous polynomial of degree $l_{k}$ in $z$ and $\bar{z}$ in $h_{k}\left(h_{k}\right.$ is defined by $\left.(2.2)\right)$. By (3.2), we get

$$
b_{q}^{j}=0, \quad \text { for any } q \in \bigcup_{k=1}^{j_{0}} Q_{k} \text { and } j=j_{0}, \ldots, d \text {. }
$$

Therefore the expansions of $g_{j_{0}}, \ldots, g_{d}$ become

$$
g_{j}=\sum_{\substack{q_{1}=\ldots=q_{j_{0}=0} \\ q_{j_{0}+1}+\ldots+q_{d} \geq 1}} b_{q}^{j}(s+i h)^{q}, \quad \text { for } j=j_{0}, \ldots, d
$$

It follows from these expansions that

$$
\frac{\partial g_{j}}{\partial s_{k}}(0)=0, \quad \text { for } j=j_{0}, \ldots, d, k=1, \ldots j_{0}
$$


Therefore the matrix $\left(\left(\partial g_{j} / \partial s_{k}\right)(0)\right)_{j, k=1, \ldots, d}$ has the following form;

$$
\left(\begin{array}{cccccc}
\frac{\partial g_{1}}{\partial s_{1}}(0) & \ldots & \frac{\partial g_{1}}{\partial s_{j_{0}}}(0) & \frac{\partial g_{1}}{\partial s_{j_{0}+1}}(0) & \ldots & \frac{\partial g_{1}}{\partial s_{d}}(0) \\
\vdots & \ddots & \vdots & \vdots & \ddots & \vdots \\
\frac{\partial g_{j_{0}-1}}{\partial s_{1}}(0) & \ldots & \frac{\partial g_{j_{0}-1}}{\partial s_{j_{0}}}(0) & \frac{\partial g_{j_{0}-1}}{\partial s_{j_{0}+1}}(0) & \ldots & \frac{\partial g_{j_{0}-1}}{\partial s_{d}}(0) \\
0 & \ldots & 0 & \frac{\partial g_{j_{0}}}{\partial s_{j_{0}+1}}(0) & \ldots & \frac{\partial g_{j_{0}}}{\partial s_{d}}(0) \\
\vdots & \ddots & \vdots & \vdots & \ddots & \vdots \\
0 & \ldots & 0 & \frac{\partial g_{d}}{\partial s_{j_{0}+1}}(0) & \ldots & \frac{\partial g_{d}}{\partial s_{d}}(0)
\end{array}\right)
$$

and the determinant of this matrix vanishes. This contradicts to the assumption and therefore completes the proof.

\section{$\S 4$. Special case of Theorem 1.1}

In this section, we consider CR mappings between hypersurfaces $M$ and $M^{\prime}$ in $\mathbb{C}^{2}$ with type $0=l$ and type $M_{0}=l^{\prime}$, respectively.

THEOREM 4.1. Let $M$ and $M^{\prime}$ be real analytic hypersurfaces in $\mathbb{C}^{2}$ with type $_{0} M=l$ and type ${ }_{0} M^{\prime}=l^{\prime}$, respectively. If a real analytic $C R$ mapping $(f, g): M \rightarrow M^{\prime}$ is not constant, then the type condition $l / l^{\prime} \in \mathbb{N}$ holds.

We drop the indices $j$ and $k$ in (2.1), (2.2) and in the expansions of $f_{j}$ and $g_{k}$.

Proof. By Theorem 1.1, we have $l \geq l^{\prime}$. If we assume $l>l^{\prime}$, then there exists $L \in \mathbb{N}$ such that $L l^{\prime}<l \leq(L+1) l^{\prime}$. Assume that $l<(L+1) l^{\prime}$. Consider the equality $\operatorname{Im} g=h^{\prime}(f, \bar{f}, \operatorname{Re} g)$, namely,

$$
\begin{aligned}
& \frac{1}{2 i} \sum_{q \geq 1} b_{q}\left[(s+i h)^{q}-(s-i h)^{q}\right] \\
&=\sum_{\substack{\nu+\mu \geq l^{\prime} \\
\nu, \mu \geq 1, \tau \geq 0}} h_{\nu, \mu, \tau}^{\prime}\left(\sum_{\alpha \geq 1, p \geq 0} a_{\alpha, p} z^{\alpha}(s+i h)^{p}\right)^{\nu}\left(\sum_{\alpha \geq 1, p \geq 0} \bar{a}_{\alpha, p} \bar{z}^{\alpha}(s-i h)^{p}\right)^{\mu} \\
& \times\left(\frac{1}{2} \sum_{q \geq 1} b_{q}\left[(s+i h)^{q}+(s-i h)^{q}\right]\right)^{\tau} .
\end{aligned}
$$

Since the minimal degree of $z$ and $\bar{z}$ on the left hand side of (4.1) equals to $l$, by observing the terms of of degree $l^{\prime}, 2 l^{\prime}, \ldots, L l^{\prime}$ in $z$ and $\bar{z}$ on the right 
hand side of (4.1) repeatedly, we obtain $a_{\alpha, p}=0$ for $\alpha=1, \ldots, L, p \geq 0$. Substituting these into (4.1) and picking up the sum of the terms of degree $l$ in $z$ and $\bar{z}$ from the resulting equality, we get

$$
\sum_{q \geq 1} b_{q} s^{q-1} h^{(l)}=0
$$

where $h^{(l)}$ is the homogeneous polynomial of degree $l$ in $z$ and $\bar{z}$ in $h$. Thus we obtain $b_{q}=0$ for $q \geq 1$, which implies $g=0$. By Lemma 2.3, we obtain $f=0$, which contradicts to the assumption. Therefore we conclude that $l=(L+1) l^{\prime}$.

\section{$\S 5$. Remark on the ratio of types}

If we consider the CR mappings between real analytic boundaries of pseudoellipsoids in $\mathbb{C}^{n}$, then the assertion of Theorem 4.1 holds even if $n \geq 3$ as in [5]. But in general, Theorem 4.1 does not hold if $n \geq 3$. In fact we have the following theorem.

Theorem 5.1. For any $A_{1}, \ldots, A_{d} \in \mathbb{Q} \backslash \mathbb{N}\left(A_{j}>1\right)$, there exist generic $C R$ submanifolds $M, M^{\prime}$ of $\mathbb{C}^{n}, n \geq 3$ with $\operatorname{dim}_{\mathbb{R}} M=\operatorname{dim}_{\mathbb{R}} M^{\prime}=2 n-d$, type $_{0} M=\left(l_{1}, \ldots, l_{d}\right)$ and type $M^{\prime}=\left(l_{1}^{\prime}, \ldots, l_{d}^{\prime}\right)$, respectively and a $C R$ mapping between them such that $A_{j}=l_{j} / l_{j}^{\prime}$, for $j=1, \ldots, d$.

Proof. Let $A_{j}=A_{j}^{1} / A_{j}^{2}$ be an irreducible fractional representation. First fix $p \in \mathbb{N}$. Put $l_{j}=2 p A_{j}^{1}$ and $l_{j}^{\prime}=2 p A_{j}^{2}$. Then there exists $q \in \mathbb{N}$ such that $q l_{j}^{\prime}<l_{j}$ for any $j$. Let

$$
\begin{aligned}
& M=\left\{\left(z_{1}, \ldots, z_{n-d}, s_{1}+i t_{1}, \ldots, s_{d}+i t_{d}\right) \in \mathbb{C}^{n} \mid\right. \\
& \left.t_{j}=\left|z_{1}\right|^{q\left(-X_{\jmath}+(n-2) L+l_{j}^{\prime}\right)}\left|z_{2}\right|^{(p+q) X_{\jmath}}\left|z_{3}\right|^{q L} \cdots\left|z_{n-d}\right|^{q L}, \text { for } j=1, \ldots, d\right\}
\end{aligned}
$$

and

$$
\begin{aligned}
& M^{\prime}=\left\{\left(z_{1}, \ldots, z_{n-d}, s_{1}+i t_{1}, \ldots, s_{d}+i t_{d}\right) \in \mathbb{C}^{n} \mid\right. \\
& \left.\quad t_{j}=\left|z_{1}\right|^{-X_{\jmath}+(n-2) L+l_{j}^{\prime}}\left|z_{2}\right|^{X_{j}}\left|z_{3}\right|^{L} \ldots\left|z_{n-d}\right|^{L}, \text { for } j=1, \cdots, d\right\},
\end{aligned}
$$

where

$$
X_{j}=\frac{l_{j}}{p}-\frac{q l_{j}^{\prime}}{p}, \quad L=\sum_{j=1}^{d}\left(l_{j}+l_{j}^{\prime}\right)
$$


Then

$$
(f, g)(z, w)=\left(z_{1}^{q}, z_{2}^{p+q}, z_{3}^{q}, \ldots, z_{n-d}^{q}, w_{1}, \ldots, w_{d}\right)
$$

is a real ánalytic CR mapping between $M$ and $M^{\prime}$. Obviously, $A_{j}=l_{j} / l_{j}^{\prime}$ for $j=1, \ldots, d$.

\section{REFERENCES}

[1] M. S. Baouendi and L. P. Rothschild, Germs of CR maps between real analytic hypersurfaces, Invent. Math., 93 (1988), 481-500.

[2] - Remarks on the generic rank of a CR mappings, J. Geom. Analysis, 2 (1992), $1-9$.

[3] T. Bloom and I. Graham, On 'type' conditions for generic real submanifolds of $\mathbb{C}^{n}$, Invent.Math., 40 (1977), 217-243.

[4] A. Boggess, CR manifolds and the tangential Cauchy-Riemann complex, CRC Press, 1991.

[5] A. Hayashimoto, Application of the $C^{\omega}$-extendability theorem for proper holomorphic mappings to the classification of the mappings between two pseudoellipsoids, Math. Japon, 44 no. 2 (1996), 221-237.

[6] M. Landucci, On the proper holomorphic equivalence for a class of pseudoconvex domains, Trans. Amer. Math. Soc., 282 (1988), 807-811.

Graduate School of Polymathematics

Nagoya University

Chikusa-ku, Nagoya, 464-01, Japan

ahayashi@math.nagoya-u.ac.jp 